\title{
Electron spin diffusion at the interface of multiferroic oxides
}

\author{
P. Zhang and M. W. Wu* \\ Hefei National Laboratory for Physical Sciences at Microscale and Department of Physics, \\ University of Science and Technology of China, Hefei, Anhui, 230026, China
}

(Dated: June 15, 2018)

\begin{abstract}
We study the spin diffusion in a two-dimensional electron gas at the interface of oxide heterostructure $\mathrm{LaAlO}_{3} / \mathrm{SrTiO}_{3}$ grown on multiferroic $\mathrm{TbMnO}_{3}$ at $15 \mathrm{~K}$ by means of the kinetic spin Bloch equation approach. The spiral magnetic moments of $\mathrm{Mn}^{3+}$ in $\mathrm{TbMnO}_{3}$ interact with the diffusing spins at the $\mathrm{LaAlO}_{3} / \mathrm{SrTiO}_{3}$ interface via the Heisenberg exchange interaction. It is demonstrated that the spin diffusion length is always finite, despite the polarization direction of the injected spins. Our study also reveals the important role played by the Coulomb scattering, which can effectively suppress the spin diffusion.
\end{abstract}

PACS numbers: 75.40.Gb, 73.20.-r, 68.47.Gh, 71.10.-w, 75.30.Et

\section{INTRODUCTION}

During the past decades, spin-based electronics has been a growing area of research due to its promising applications. ${ }^{1-5}$ Among various electron systems, the two-dimensional electron gas (2DEG) has been widely studied in spin injection, spin relaxation, spin transport and spin Hall effect, etc.. ${ }^{4-17}$ Most of the 2DEG systems are achieved in the semiconductor quantum wells or heterostructures. ${ }^{18}$ The strict $2 \mathrm{DEG}$, e.g., the one existing in a graphene monolayer, also attracts much attention recently. ${ }^{11-14,19-21}$ Besides, the 2DEG has also been experimentally realized at the interface of insulating oxides such as $\mathrm{LaAlO}_{3} / \mathrm{SrTiO}_{3},{ }^{22-24}$ opening the way for the oxide-based nanoelectronics. Starting from this oxide heterostructure, very recently Jia and Berakdar proposed a trilayer system for new functionalities, which is grown as $\mathrm{LaAlO}_{3} / \mathrm{SrTiO}_{3} / \mathrm{TbMnO}_{3}$ along the $c$-axis of orthorhombic $\mathrm{TbMnO}_{3} \cdot{ }^{25-28}$ As a multiferroic material, $\mathrm{TbMnO}_{3}$ can exhibit both magnetic and electric orders simultaneously and provide a unique opportunity to exploit the multifunctionality of a single material. ${ }^{29-31} \mathrm{SrTiO}_{3}$ in the middle of the trilayer system is a few layers thick so that the 2DEG at the interface of $\mathrm{LaAlO}_{3} / \mathrm{SrTiO}_{3}$ can be affected by the magnetic order in $\mathrm{TbMnO}_{3}$. Based on this structure, Jia and Berakdar predicted the appearance of a persistent spin current in the 2DEG due to the spiral geometry of the local magnetic order. ${ }^{25}$ They also proposed a flash memory model based on the claim that the injected spins in the 2DEG have no decay of spin polarization along the $a$-axis when travelling along the $b$-axis. ${ }^{26}$ This claim was inferred ${ }^{26}$ from the fact that the spin polarization along the [110] direction does not decay in (001) GaAs quantum-well-based 2DEG with identical Rashba ${ }^{32}$ and Dresselhaus $^{33}$ spin-orbit coupling strengths, in the $a b$ sence of any external magnetic field. ${ }^{34-37}$ Nevertheless, this claim is questionable in the 2DEG formed at the interface of the oxide, because the local magnetic moments serve as a static magnetic field in the Voigt configuration in the spiral frame ${ }^{26}$ and, as pointed out by Weng and
$\mathrm{Wu},{ }^{38}$ a magnetic field in the Voigt configuration can effectively suppress spin diffusion/transport even when the D'yakonov-Perel' spin-orbit coupling ${ }^{39}$ is absent. ${ }^{38,40}$ In this work, we investigate the spin diffusion along the $b$-axis at the interface of $\mathrm{LaAlO}_{3} / \mathrm{SrTiO}_{3}$ closely above $\mathrm{TbMnO}_{3}$ by means of the kinetic spin Bloch equation (KSBE) approach. ${ }^{5}$ It is found that the spin diffusion length is finite in spite of the spin polarization direction and the Coulomb scattering makes marked effect on the spin relaxation in diffusion .

This paper is organized as follows. In Sec. II, we give the Hamiltonian of the 2DEG in the collinear frame with $\hat{\boldsymbol{\sigma}}_{z}$ parallel to the $c$-axis, and in the spiral frame with $\hat{\boldsymbol{\sigma}}_{z}$ parallel to the local magnetic moment, respectively. We then present the KSBEs in the spiral frame. In Sec. III we study the spin diffusion based on the KSBEs. We summarize in Sec. IV.

\section{HAMILTONIAN AND KSBES}

The 2DEG formed at the $\mathrm{LaAlO}_{3} / \mathrm{SrTiO}_{3}$ interface of $\mathrm{LaAlO}_{3} / \mathrm{SrTiO}_{3} / \mathrm{TbMnO}_{3}$ compounds is schematically shown in Ref. 25. We assume it is confined by an infinitely deep square potential well with width $a=3 \mathrm{~nm} .{ }^{22}$ The coordinate system is set as $\hat{\mathbf{x}}=\hat{\mathbf{b}}, \hat{\mathbf{y}}=-\hat{\mathbf{a}}$ and $\hat{\mathbf{z}}=\hat{\mathbf{c}}$. When the temperature $T$ is lower than the ferroelectric Curie temperature $T_{c}(\sim 27 \mathrm{~K}), \mathrm{Mn}^{3+}$ magnetic moments in $\mathrm{TbMnO}_{3}$ form a spiral order with the local magnetic moment $\mathbf{M}(\mathbf{r})=M_{x} \sin (\mathbf{q} \cdot \mathbf{r}) \hat{\mathbf{x}}+M_{z} \cos (\mathbf{q} \cdot \mathbf{r}) \hat{\mathbf{z}}$, where $\mathbf{q}=\left(0.27 \times \frac{2 \pi}{b}, 0, \frac{2 \pi}{c}\right)$ is the modulation vector. ${ }^{29,41-43}$ $b=0.586 \mathrm{~nm}$ and $c=0.749 \mathrm{~nm}$ are the lattice parameters of $\mathrm{TbMnO}_{3} .{ }^{29}$ In reality $M_{x} \neq M_{z}$ (e.g., $M_{x} / M_{z} \approx 1.4$ at $T=15 \mathrm{~K}),{ }^{29,41-43}$ however for simplicity we take $M_{x} \approx M_{z}=M$ following Jia and Berakdar. ${ }^{25,26} \mathrm{It}$ is assumed that the 2DEG only interacts with the local magnetic momentums of $\mathrm{Mn}^{3+}$ on the surface of $\mathrm{TbMnO}_{3}$ (i.e., the plane $z=0$ ) via the Heisenberg exchange interaction. ${ }^{25,26}$ As a result, the Hamiltonian of 
the $2 \mathrm{DEG}$ reads ${ }^{25,26}$

$$
H=\frac{\mathbf{P}^{2}}{2 m^{*}}+J \hat{\mathbf{n}}_{\mathbf{r}} \cdot \boldsymbol{\sigma},
$$

with the first term on the right-hand side of the equation representing the kinetic energy and the second term, the exchange interaction. $m^{*}$ is the effective electron mass set to be $10 m_{e}\left(m_{e}\right.$ is the free-electron mass), ${ }^{25,26} J$ stands for the coupling strength, $\hat{\mathbf{n}}_{\mathbf{r}}=$ $\left(\sin \left(q_{x} x\right), 0, \cos \left(q_{x} x\right)\right)$ denotes the unit vector along the local magnetic moment located on $z=0$ plane, $\mathbf{P}$ is the momentum operater and $\boldsymbol{\sigma}$ is the vector of the Pauli matrices. Performing a local rotation around the $y$-axis in the spin space as $\tilde{H}=U_{g}^{\dagger}(x) H U_{g}(x)$ with $U_{g}(x)=$ $e^{-i q_{x} x \sigma_{y} / 2}$, one obtains in the spiral frame ${ }^{25,26}$

$$
\tilde{H}=\frac{1}{2 m^{*}}\left[\left(P_{x}-\frac{\hbar q_{x} \sigma_{y}}{2}\right)^{2}+P_{y}^{2}\right]+J \sigma_{z} .
$$

This Hamiltonian can be written in the momentum space as

$$
\tilde{H}=\frac{\hbar^{2} \mathbf{k}^{2}}{2 m^{*}}+\mathbf{h}_{\mathbf{k}} \cdot \boldsymbol{\sigma},
$$

in which

$$
\mathbf{h}_{\mathbf{k}}=\left(0, \frac{\hbar^{2} q_{x}}{2 m^{*}} k_{x}, J\right)
$$

and a uniform energy displacement $\varepsilon_{0}=\frac{\hbar^{2} q_{x}^{2}}{8 m^{*}}$ is omitted. The position-dependent $3 \times 3$ orthogonal rotation matrix $R(x)$, which obeys $U_{g}^{\dagger}(x) \boldsymbol{\sigma} U_{g}(x)=R(x) \boldsymbol{\sigma}$, reads

$$
R(x)=\left(\begin{array}{ccc}
\cos \left(q_{x} x\right) & 0 & \sin \left(q_{x} x\right) \\
0 & 1 & 0 \\
-\sin \left(q_{x} x\right) & 0 & \cos \left(q_{x} x\right)
\end{array}\right) .
$$

$R^{T}(x)$ transforms any spin-vector in the spiral frame back to the collinear frame. Our study is performed by first solving the KSBEs in the spiral frame and then obtaining the spin diffusion properties in the collinear frame with the aid of $R^{T}(x)$.

In the study, polarized spins are injected at $x=0$ and diffuse along the $x$-axis. The system is uniform along the fixed $y$-axis in both frames. In the spiral frame, the KSBEs read ${ }^{5}$

$$
\begin{aligned}
& \frac{\partial \rho_{\mathbf{k}}(x, t)}{\partial t}+\frac{e}{\hbar} \frac{\partial \Psi(x, t)}{\partial x} \frac{\partial \rho_{\mathbf{k}}(x, t)}{\partial k_{x}}+\frac{\hbar k_{x}}{m^{*}} \frac{\partial \rho_{\mathbf{k}}(x, t)}{\partial x} \\
& +\frac{i}{\hbar}\left[\mathbf{h}_{\mathbf{k}} \cdot \boldsymbol{\sigma}, \rho_{\mathbf{k}}(x, t)\right]+\left.\frac{\partial \rho_{\mathbf{k}}(x, t)}{\partial t}\right|_{\text {scat }}=0,
\end{aligned}
$$

where $\rho_{\mathbf{k}}(x, t)$ are the single-particle density matrices of electrons with wave-vector $\mathbf{k}$ at position $x$ and time $t$. $\frac{e}{\hbar} \frac{\partial \Psi(x, t)}{\partial x} \frac{\partial \rho_{\mathbf{k}}(x, t)}{\partial k_{x}}$ are the driving terms with the electric potential satisfying the Poisson equation $\nabla_{x}^{2} \Psi(x, t)=$ $e\left[N_{e}(x, t)-N_{0}\right] /\left(a \kappa_{0} \varepsilon_{0}\right)$, where $N_{e}(x, t)=\sum_{\mathbf{k}} \operatorname{Tr}\left[\rho_{\mathbf{k}}(x, t)\right]$ stands for the electron density at position $x$ and time $t$, $N_{0}$ is the background positive charge density and $\kappa_{0} \approx 24$ is the relative static dielectric constant. ${ }^{44,45}$ In the absence of the external electric field, the driving term is negligible. $\frac{\hbar k_{x}}{m^{*}} \frac{\partial \rho_{\mathbf{k}}(x, t)}{\partial x}, \frac{i}{\hbar}\left[\mathbf{h}_{\mathbf{k}} \cdot \boldsymbol{\sigma}, \rho_{\mathbf{k}}(x, t)\right]$ and $\left.\frac{\partial \rho_{\mathbf{k}}(x, t)}{\partial t}\right|_{\text {scat }}$ in Eq. (6) are the diffusion, coherent and scattering terms, respectively. We lack detailed information of the electron-phonon and electron-magnon scatterings in this material, except that the electron-phonon scattering in $\mathrm{SrTiO}_{3}$ was suggested to be typically very weak. ${ }^{24}$ However, as we are interested only in the low temperature behavior (we take $T=15 \mathrm{~K}<T_{c}$ in this paper), both scatterings are neglected. The electron-impurity scattering is also neglected in the high-mobility 2DEG (for electron density $N_{e} \sim 10^{13} \mathrm{~cm}^{-2}$ and mobility $\sim 10^{4} \mathrm{~cm}^{2} /(\mathrm{V} \cdot \mathrm{s}),{ }^{22}$ the impurity density is calculated to be $\sim 10^{8} \mathrm{~cm}^{-2}$, corresponding to which the electron-impurity scattering is found to be negligible). The scattering left in our consideration is the electron-electron Coulomb scattering, which has been proved to be important in spin relaxation and spin transport in semiconductors. ${ }^{5,46-48}$ The detailed expression of the Coulomb scattering can be found in Ref. 49. By solving the KSBEs one obtains the spin diffusion properties from $\rho_{\mathbf{k}}(x,+\infty)$, the steady-state distribution of density matrices.

\section{SPIN DIFFUSION}

\section{A. Spin relaxation in time domain}

Equation (4) is analogous to the spin-orbit coupling in (001) GaAs quantum-well-based 2DEG with identical Rashba and Dresselhaus spin-orbit coupling strengths (only the linear Dresselhaus term is considered) when $J$ is set to zero. There, when the $x$-axis is set along [110], the spin-orbit coupling reads $\tilde{\mathbf{h}}_{\mathbf{k}}=\left(0,2 \gamma k_{x}, 0\right)$, with $\gamma$ being the coefficient of the Rashba (or Dresselhaus) spinorbit coupling. ${ }^{35,36,48,50}$ Therefore, if the spin polarization is along the $y$-axis, there is no spin precession and hence the spin relaxation is suppressed. ${ }^{35,36,48,50}$ However, the exchange interaction in oxide provides a static magnetic field $J$ along the $z$-axis in the spiral frame, as shown in Eq. (4). This field rotates the spin polarization away from the $y$-axis. The spin polarization away from the $y$-axis feels the inhomogeneous broadening, ${ }^{5,46}$ i.e., the momentum-dependent spin-orbit coupling from the $y$-component of $\mathbf{h}_{\mathbf{k}}$, which, together with any scattering, leads to irreversible spin relaxation. $5,46,48$ Moreover, the effective magnetic field mixes spin relaxations along the $x-z$ plane to the one along the $y$-axis, similar to the case based on semiconductor quantum wells. ${ }^{36,48}$ Therefore, the spin relaxation time along the $y$-axis is not infinite as claimed by Jia and Berakdar, ${ }^{26}$ neither does the spin relaxation along other directions. The above scenario also happens to (110) GaAs quantum wells. The effective magnetic field induced by the Dresselhaus spin-orbit coupling is oriented along the quantum-well growth direction when only the lowest subband is relevant, which 
leads to an infinite spin relaxation time when the spin polarization is along that direction. However, by applying a static magnetic field in the quantum-well plane, the spin relaxation time becomes finite. ${ }^{51,52}$

To be quantitative, we numerically solve the KSBEs in time domain ${ }^{5,38,47-49}$ with the Coulomb scattering explicitly included. In Fig. 1, the time evolution of spin polarization (with an initial value $5 \%$ ) is plotted. It is clearly shown that when the initial spin polarization $\hat{\mathbf{n}}$ is along $\hat{\mathbf{y}}$, the spin polarization relaxes when $J \neq 0$. However, for other spin polarization directions such as $\hat{\mathbf{z}}$, spin relaxes even when $J=0$.

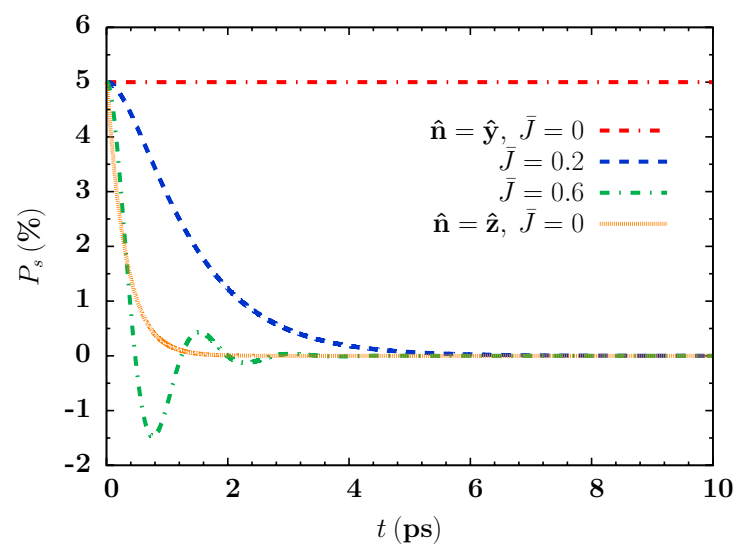

FIG. 1: (Color online) Time evolution of spin polarization along different directions under different $J$ (in the figure $\bar{J}=$ $\left.\frac{m^{*}}{\pi N_{e} \hbar^{2}} J\right)$.

\section{B. Spin diffusion: analytical and numerical study}

In this section we investigate the spin diffusion of the 2DEG. We first consider a simplified case without the scattering for which the KSBEs can be solved analytically. Then we numerically solve the KSBEs in the presence of the Coulomb scattering.

When the scattering terms are excluded (and the driving terms are absent), the KSBEs can be solved in the steady state as

$$
\rho_{\mathbf{k}}(x,+\infty)=e^{-i \boldsymbol{\omega}_{\mathbf{k}} \cdot \boldsymbol{\sigma} x / 2} \rho_{\mathbf{k}}(0,+\infty) e^{i \boldsymbol{\omega}_{\mathbf{k}} \cdot \boldsymbol{\sigma} x / 2}
$$

with

$$
\boldsymbol{\omega}_{\mathbf{k}}=\frac{2 m^{*}}{\hbar^{2} k_{x}} \mathbf{h}_{\mathbf{k}}=\left(0, q_{x}, \frac{2 m^{*}}{\hbar^{2} k_{x}} J\right)
$$

depicting the spin precession frequency in spatial domain. It has been shown that the inhomogeneous broadening in spin diffusion/transport is determined by $\boldsymbol{\omega}_{\mathbf{k}}$ rather than $\mathbf{h}_{\mathbf{k}}$ in the time domain, $, 5,37,38,40,48$ with $k_{x}$ arising from the diffusion term. It becomes evident that $J$, playing the role of a static magnetic field in the time domain, now leads to the inhomogeneous broadening due to $k_{x}^{-1}$ and hence a finite spin injection length. Similar effect
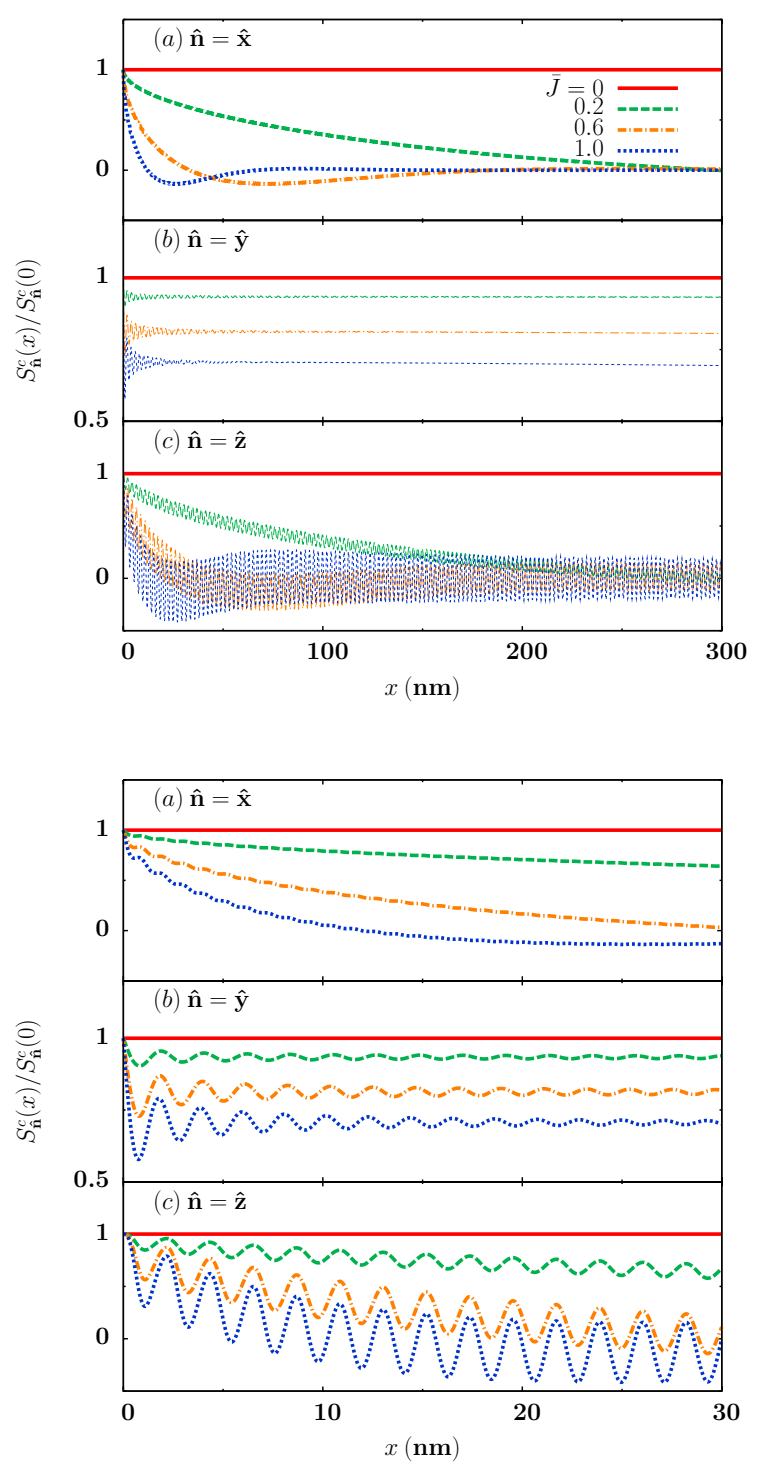

FIG. 2: (Color online) $S_{\hat{\mathbf{n}}}^{c}(x) / S_{\hat{\mathbf{n}}}^{c}(0)$ vs. $x$ for different $\hat{\mathbf{n}}$ and $\bar{J}$. (a) and (d): $\hat{\mathbf{n}}=\hat{\mathbf{x}} ;(\mathrm{b})$ and $(\mathrm{e}): \hat{\mathbf{n}}=\hat{\mathbf{y}} ;(\mathrm{c})$ and $(\mathrm{f}): \hat{\mathbf{n}}=\hat{\mathbf{z}}$. (a)-(c) are plotted in a large scale of $x$ while (d)-(f) are in a small scale.

was first predicted theoretically by Weng and Wu back in 2002 (Ref. 38) and realized experimentally in bulk silicon. ${ }^{40,53}$ Moreover, due to the presence of $q_{x}$ in Eq. (8), the spin diffusion length is finite even when the injected spins are polarized along the $z$-axis.

The spatial distribution of spin polarization is obtained from the density matrices given by Eq. (7). We consider the states with $k_{x}>0$ because only these states can propagate along the $x$-axis and the states with $k_{x}<0$ are not spin-polarized in the absence of scattering. ${ }^{48}$ Therefore the density matrices with $k_{x}>0$ at $x=0$ are fixed as boundary conditions. The spin polarization $\mathbf{S}_{\mathbf{k}}(x) \equiv$ $\operatorname{Tr}\left[\rho_{\mathbf{k}}(x,+\infty) \boldsymbol{\sigma}\right]$ is calculated to be $\mathbf{S}_{\mathbf{k}}(x)=F_{\mathbf{k}}(x) \mathbf{S}_{\mathbf{k}}(0)$, 
with

$$
F_{\mathbf{k}}(x)=\left(\begin{array}{ccc}
\cos \left(\omega_{\mathbf{k}} x\right) & -\hat{h}_{\mathbf{k}, z} \sin \left(\omega_{\mathbf{k}} x\right) & \hat{h}_{\mathbf{k}, y} \sin \left(\omega_{\mathbf{k}} x\right) \\
\hat{h}_{\mathbf{k}, z} \sin \left(\omega_{\mathbf{k}} x\right) & \hat{h}_{\mathbf{k}, y}^{2}+\hat{h}_{\mathbf{k}, z}^{2} \cos \left(\omega_{\mathbf{k}} x\right) & \hat{h}_{\mathbf{k}, y} \hat{h}_{\mathbf{k}, z}\left[1-\cos \left(\omega_{\mathbf{k}} x\right)\right] \\
-\hat{h}_{\mathbf{k}, y} \sin \left(\omega_{\mathbf{k}} x\right) & \hat{h}_{\mathbf{k}, y} h_{\mathbf{k}, z}\left[1-\cos \left(\omega_{\mathbf{k}} x\right)\right] & \hat{h}_{\mathbf{k}, z}^{2}+\hat{h}_{\mathbf{k}, y}^{2} \cos \left(\omega_{\mathbf{k}} x\right)
\end{array}\right)
$$

in which $\omega_{\mathbf{k}}=\left|\boldsymbol{\omega}_{\mathbf{k}}\right|, \hat{h}_{\mathbf{k}, y(z)}=h_{\mathbf{k}, y(z)} /\left|\mathbf{h}_{\mathbf{k}}\right|$ and $\mathbf{S}_{\mathbf{k}}(0)=$ $\operatorname{Tr}\left[\rho_{\mathbf{k}}(0,0) \boldsymbol{\sigma}\right]$. The total spin signal in the spiral frame is

$$
\mathbf{S}(x)=\sum_{k_{x}>0} \mathbf{S}_{\mathbf{k}}(x)=\sum_{k_{x}>0} F_{\mathbf{k}}(x) \mathbf{S}_{\mathbf{k}}(0),
$$

and in the collinear frame reads

$$
\mathbf{S}^{c}(x)=R^{T}(x) \mathbf{S}(x) .
$$

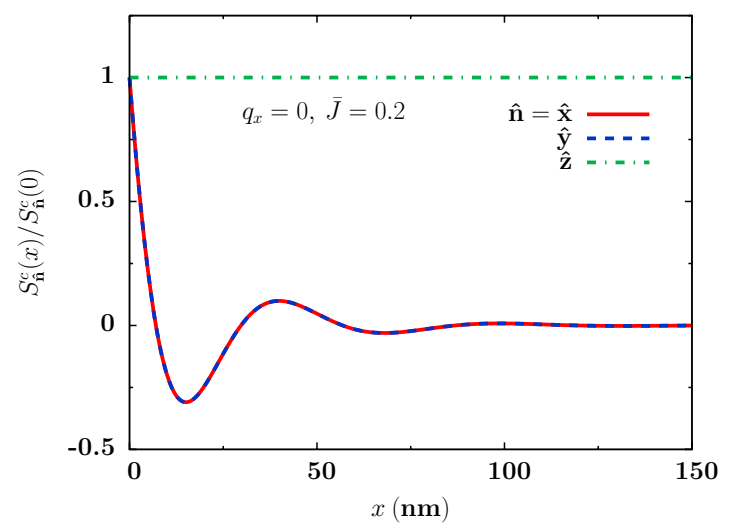

FIG. 3: (Color online) $S_{\hat{\mathbf{n}}}^{c}(x) / S_{\hat{\mathbf{n}}}^{c}(0)$ vs. $x$ for different $\hat{\mathbf{n}}$ when $q_{x}$ is set to be zero. $\bar{J}=0.2$.

In Fig. 2, the projection of spin signal $\mathbf{S}^{c}(x)$ on the injected spin polarization direction $\hat{\mathbf{n}}, S_{\hat{\mathbf{n}}}^{c}(x) \equiv \mathbf{S}^{c}(x) \cdot \hat{\mathbf{n}}$, calculated from Eq. (11) is plotted against $x$ with different $J$ [the data are scaled by $\left.S_{\hat{\mathbf{n}}}^{c}(0)\right]$. In the computation, we set $\mathbf{S}_{\mathbf{k}}(0)=\left[f\left(\varepsilon_{\mathbf{k}}-\mu_{+}\right)-f\left(\varepsilon_{\mathbf{k}}-\mu_{-}\right)\right] \hat{\mathbf{n}} .{ }^{37,40,48}$ $f\left(\varepsilon_{\mathbf{k}}-\mu_{\xi}\right)$ with $\varepsilon_{\mathbf{k}}=\hbar^{2} k^{2} / 2 m^{*}$ is the Fermi distribution under temperature $T$. The chemical potential $\mu_{\xi}$ is determined by $N_{e}$, the electron density of the 2DEG, and $P_{s}$, the polarization of injected spins, at $x=0$ via the relation $\sum_{\mathbf{k}} f\left(\varepsilon_{\mathbf{k}}-\mu_{\xi}\right)=0.5\left(1+\xi P_{s}\right) N_{e} .^{37,40,48}$ We set $N_{e}=10^{13} \mathrm{~cm}^{-2}$ (Ref. 22) and $P_{s}=5 \%$. $\hat{\mathbf{n}}$ is set as $\hat{\mathbf{x}}$, $\hat{\mathbf{y}}$ and $\hat{\mathbf{z}}$. It is shown that when $J \neq 0$, the spin polarization decays during spin diffusion. When $J$ increases, the decay becomes faster. It is noted that $S_{\hat{\mathbf{n}}}^{c}(x)$ relaxes to a finite value for $\hat{\mathbf{n}}=\hat{\mathbf{y}}$ in the absence of scattering. However, when the scattering is included, the spin polarization will completely relax to zero during spin diffusion (this will be shown later). Moreover, the spatial distribution of spin polarization shows high-frequency oscillations when $J \neq 0$, as detailed in Figs. 2(d)-(f) in a smaller scale of $x$. The frequency of these oscillations is comparable to the rotation frequency of the spiral magnetic moments $q_{x}$ and insensitive to the exchange coupling strength. For comparison, we recalculate $S_{\hat{\mathbf{n}}}^{c}(x)$ by replacing the spiral order with the ferromagnetic order, i.e., aligning all the magnetic moments uniformly along the $z$-axis. Hence $q_{x}=0$. The corresponding results are shown in Fig. 3, where the high-frequency oscillations disappear and the spin polarization along the $z$-axis does not decay due to the absence of the inhomogeneous broadening.

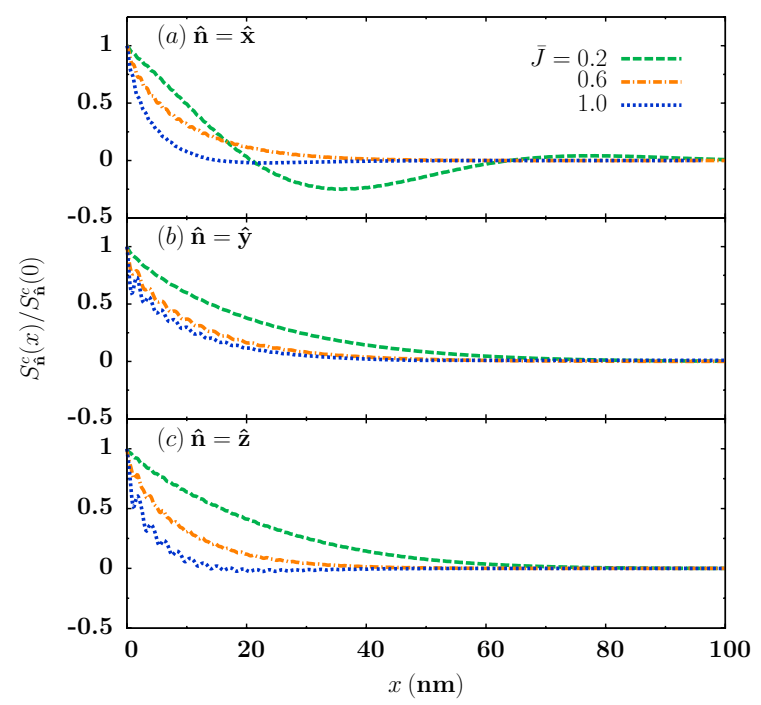

FIG. 4: (Color online) $S_{\hat{\mathbf{n}}}^{c}(x) / S_{\hat{\mathbf{n}}}^{c}(0) v s . x$ for different $\hat{\mathbf{n}}$ and $\bar{J}$. Here $S_{\hat{\mathbf{n}}}^{c}(x)$ are obtained by numerically solving the KSBEs in the presence of the Coulomb scattering.

It is noted that the above decay of spin polarization along the spin diffusion in the absence of scattering is due to the interference effect. ${ }^{38}$ Now we numerically solve the full KSBEs with the Coulomb scattering explicitly included, facilitated with the double-side injection boundary conditions. ${ }^{5,21,37,40}$ For the cases with $J \neq 0$, we plot $S_{\hat{\mathbf{n}}}^{c}$ as a function of position $x$ in Fig. 4. It is shown that the Coulomb scattering effectively leads to the spin relaxation along the spin diffusion. By comparing Figs. 4 and 2 , it is observed that with the scattering, the spin diffusion is suppressed along all directions. ${ }^{40}$ Especially, the 
spin polarization along the $y$-axis relaxes to zero completely during the spin diffusion. It is also noted that the amplitude of the high-frequency oscillations shown in Fig. 2 becomes much smaller.

\section{CONCLUSION}

In conclusion, we have studied the spin diffusion in a 2DEG at the interface of oxide heterostructure $\mathrm{LaAlO}_{3} / \mathrm{SrTiO}_{3}$ closely above the multiferroic $\mathrm{TbMnO}_{3}$ by means of the KSBE approach. At low temperature $(<27 \mathrm{~K}), \mathrm{Mn}^{3+}$ magnetic moments in $\mathrm{TbMnO}_{3}$ form a spiral order and couple with the $2 \mathrm{DEG}$ via the Heisenberg exchange interaction. It is found that in the presence of the spiral magnetic order, in contrast to the claims in the literature, the spin polarization decays during spin diffusion, despite the polarization direction of injected spins. We also report that the electron-electron Coulomb scattering suppresses spin diffusion effectively at low temperature.

\section{Acknowledgments}

We would like to thank J. Berakdar for valuable discussions. This work was supported by the National Natural Science Foundation of China under Grant No. 10725417 and the Knowledge Innovation Project of Chinese Academy of Sciences.
* Author to whom correspondence should be addressed; Electronic address: mwwu@ustc.edu.cn.

1 Semiconductor Spintronics and Quantum Computation, edited by D. D. Awschalom, D. Loss, and N. Samarth (Sprinter, Berlin, 2002).

2 I. Žutić, J. Fabian, and S. D. Sarma, Rev. Mod. Phys. 76, 323 (2004).

3 Spin Physics in Semiconductors, ed. by M. I. D'yakonov (Springer, Berlin, 2008).

4 J. Fabian, A. Matos-Abiague, C. Ertler, P. Stano, and I. Žutić, Acta Phys. Slov. 57, 565 (2007).

5 M. W. Wu, J. H. Jiang, and M. Q. Weng, Phys. Rep. 493, $61(2010)$.

6 Y. Ohno, D. K. Young, B. Beschoten, F. Matsukura, H. Ohno, and D. D. Awschalom, Nature (London) 402, 790 (1999).

7 B. T. Jonker, Y. D. Park, B. R. Bennett, H. D. Cheong, G. Kioseoglou, and A. Petrou, Phys. Rev. B 62, 8180 (2000).

8 Y. K. Kato, R. C. Myers, A. C. Gossard, and D. D. Awschalom, Science 306, 1910 (2004).

9 J. Wunderlich, B. Kaestner, J. Sinova, and T. Jungwirth, Phys. Rev. Lett. 94, 047204 (2005).

10 S. O. Valenzuela and M. Tinkham, Nature 442, 176 (2006).

11 M. Ohishi, M. Shiraishi, R. Nouchi, T. Nozaki, T. Shinjo, and Y. Suzuki, Jpn. J. Appl. Phys. 46, L605 (2007).

12 N. Tombros, C. Józsa, M. Popinciuc, H. T. Jonkman, and B. J. van Wees, Nature (London) 448, 571 (2007).

13 A. H. C. Neto, F. Guinea, N. M. R. Peres, K. S. Novoselov, and A. K. Geim, Rev. Mod. Phys. 81, 109 (2009).

14 D. S. L. Abergel, V. Apalkov, J. Berashevich, K. Ziegler, and T. Chakraborty, Adv. Phys. 59, 261 (2010).

15 Z. Chen, S. G. Carter, R. Bratschitsch, and S. T. Cundiff, Physica E 42, 1803 (2010).

16 M. M. Glazov, E. Ya. Sherman, and V. K. Dugaev, Physica E 42, 2157 (2010).

17 T. Korn, Phys. Rep. 494, 415 (2010).

18 J. H. Davies, The physics of low-dimensional semiconductors: an introduction (Cambridge University Press, Cambridge, 1998).

19 A. K. Geim and K. S. Novelia, Nature Mater. 6, 183 (2007).

${ }^{20}$ Y. Zhou and M. W. Wu, Phys. Rev. B 82, 085304 (2010).

${ }^{21}$ P. Zhang and M. W. Wu, arXiv: 1012.0973.

22 S. Thiel, G. Hammerl, A. Schmehl, C. W. Schnerder, and
J. Mannhart, Science 313, 1942 (2006).

23 A. Ohtomo and H. Y. Hwang, Nature (London) 427, 423 (2004)

24 M. Huijben, G. Rijnders, D. H. A. Blank, S. Bals, S. V. Aert, J. Verbeeck, G. V. Tendeloo, A. Brinkman, and H. Hilgenkamp, Nature 5, 556 (2006).

${ }^{25}$ C. Jia and J. Berakdar, Phys. Rev. B 80, 014432 (2009).

26 C. Jia and J. Berakdar, Appl. Phys. Lett. 95, 012105 (2009).

27 C. Jia and J. Berakdar, arXiv:1012.4865.

28 C. Jia and J. Berakdar, Phys. Rev. B 83, 045309 (2011).

29 M. Kenzelmann, A. B. Harris, S. Jonas, C. Broholm, J. Schefer, S. B. Kim, C. L. Zhang, S. W. Cheong, O. P. Vajk, and J. W. Lynn, Phys. Rev. Lett. 95, 087206 (2005).

30 M. Gajek, M. Bibes, S. Fusil, K. Bouzehouane, J. Fontcuberta, A. Barthélémy, and A. Fert, Nat. Mater. 6, 296 (2007).

31 N. Hur, S. Park, P. A. Sharma, J. S. Ahn, S. Guha, and S. W. Cheong, Nature 429, 392 (2004).

32 Y. A. Bychkov and E. I. Rashba, J. Phys. C 17, 6039 (1984).

33 G. Dresselhaus, Phys. Rev. 100, 580 (1955).

34 N. S. Averkiev, L. E. Golub, and M. Willander, J. Phys.: Condens. Matt. 14, R271 (2002).

35 J. Schliemann, J. C. Egues, and D. Loss, Phys. Rev. Lett. 90, 146801 (2003).

36 D. Stich, J. H. Jiang, T. Korn, R. Schulz, D. Schuh, W. Wegscheider, M. W. Wu, and C. Schüller, Phys. Rev. B 76, 073309 (2007).

37 J. L. Cheng, M. W. Wu, and I. C. da Cunha Lima, Phys. Rev. B 75, 205328 (2007).

38 M. Q. Weng and M. W. Wu, Phys. Rev. B 66, 235109 (2002).

39 M. I. D'yakonov and V. I. Perel', Zh. Éksp. Teor. Fiz. 60, 1954 (1971) [Sov. Phys. JETP 33, 1053 (1971)].

40 P. Zhang and M. W. Wu, Phys. Rev. B 79, 075303 (2009).

41 Y. Yamasaki, H. Sagayama, T. Goto, M. Matsuura, K. Hirota, T. Arima, and Y. Tokura, Phys. Rev. Lett. 98, 147204 (2007).

42 T. Arima, A. Tokunaga, T. Goto, H. Kimura, Y. Noda, and Y. Tokura, Phys. Rev. Lett. 96, 097202 (2007).

43 A. Malashevich and D. Vanderbilt, Phys. Rev. Lett. 101, 037210 (2008). 
44 T. Konaka, M. Sato, H. Asano, and S. Kubo, J. Supercond., 4, 283 (1991).

45 Y. Kozuka, M. Kim, C. Bell, B. G. Kim, Y. Hikita, and H. Y. Hwang, Nature 462, 487 (2009).

46 M. W. Wu and C. Z. Ning, Eur. Phys. J. B 18, 373 (2000).

47 M. Q. Weng and M. W. Wu, Phys. Rev. B 68, 075312 (2003).

48 J. L. Cheng and M. W. Wu, J. Appl. Phys. 99, 083704 (2006).

49 M. Q. Weng, M. W. Wu, and L. Jiang, Phys. Rev. B 69, 245320 (2004).
50 N. S. Averkiev and L. E. Golub, Phys. Rev. B 60, 15582 (1999).

51 M. W. Wu and M. Kuwata-Gonokami, Solid State Commun. 121, 509 (2002).

52 S. Döhrmann, D. Hägele, J. Rudolph, M. Bichler, D. Schuh, and M. Oestreich, Phys. Rev. Lett. 93, 147405 (2004).

53 I. Appelbaum, B. Huang, and D. J. Monsma, Nature (London) 447, 295 (2007). 